\title{
INFLUENCE OF PLASMA TREATMENT ON PROPERTIES OF WAXED PAPERS
}

\author{
Igor Karlovits (D), Gregor Lavrič (iD) \\ Pulp and Paper Institute, Ljubljana, Slovenia
}

\begin{abstract}
Barrier coatings of fibrous materials are of great interest for the packaging paper producers as increased resistance to oils, grease and other agents with the paper packaging increases the usability of the product. The barrier coatings have to withhold several parameters regarding resistance for the penetration of various agents, runnability on the machines regarding mechanical properties and also printability futures to enhance the packaging. Different sacks, bags and other products are mainly produced by coating the surface of the paper with the appropriate coatings which can be petroleum-based waxes, polymers solutions or pigments. By applying the barrier coatings the surface topography and porosity as well the surface chemistry is changed as the used polymers, waxes and other products which fill the fibrous network of the packaging change the macrostructure and induce different chemical compounds on the surface. These products are mainly printed with flexo technology We have checked the grease resistance with the KIT test (Tappi T 559), dynamic contact angle change, the penetration of liquids and the surface coverage of plasma treated waxed and base papers. Our results indicate improved printability and liquid penetration but also the lowering of grease resistance after plasma treatment. The treatment is possible but further adjustments regarding plasma power and time of treatment are needed to find the optimal parameters for different kind of barrier coated papers.
\end{abstract}

Key words: waxed paper, plasma, dynamic contact angle, liquid penetration dynamics

\section{INTRODUCTION}

The waxed paper provides an economical choice for primary food packaging not only because of their versatility but also because of their safety as tasteless, odourless, non-toxic and relatively inert materials. Their widespread use in conventional packaging applications includes sandwich wraps and papers, bags, food trays, folding cartons, overwraps and many other types of packaging materials (Kit, 2010). The waxing material used is predominantly pure paraffin, often modified by the addition of microcrystalline wax and resins, particularly polyethene. There are two general kinds of waxed paper: dry waxed, in which sheet is saturated with wax with none being left on the surface; and wet waxed, in which some wax is left as a film on the surface of the sheet (National Research Council, 1959). Recently different trends induced several changes on the market, one from the change of type of the barrier substance (removal of paraffin - change to biopolymer coatings), the need for packaging attractiveness improvement with multi-colour printing and food safety issues regarding contact with food and the migration of inks or other substances through the waxed paper. In her Bachelor theses, Sedlacekova (2017) made a comparison of different wrapping materials where the waxed paper has some beneficial properties as no composite layers are needed and the logistics point of view, the wax layer on the paper or paperboard improves the strength of the package making it unproblematic to stack. From the downside, waxed papers are harder to recycle because the wax layer should be separated (Coles et al., 2011). Also, trends of using recycled paper as a base paper instead of using virgin fibre cellulose materials (due to their availability and high prices) are having market potential regarding the decline of the reuse of the recycled materials for the newspaper or other graphic communication materials. Regarding food safety, the wrapping papers are mostly printed with water-based inks (with no VOCs or UV components) which induce that flexo printing is a dominant technology at the moment for decorating these kinds of papers. The waxed papers due to their surface treatment pose a challenge for water-based inks due to some natural incompatibilities, but the use of different surface modification methods like plasma or corona treatment there is a way to adhere the printing ink on the surface. Plasma is known as the fourth state of matter, it's a high-energy gas consisting of a mixture of ions, free radicals and photons. The energy by this particular gas allows changing the surfaces without affecting mass properties (bulk proprieties). Plasma flow is generated by submitting any gas, even simple air, to an electric field. This causes acceleration of free electrons that collide with gas atoms and molecules; depending on the type of collision and electron energy, ions or excited species are originated. Chemically reactive species are able to modify the surfaces with which they come into contact, by removing molecules (cleaning) or by adding functional groups (etching) (Brunelli, 2013). Researchers mainly tried using plasma for surface functionalization, but also some research work is found 
regarding surface modification of cellulose-based or cellulose combined materials. It is well known that atmospheric pressure plasma activation in the air creates polar oxygen molecular groups on the surface, and thus increases the surface energy. Changes in the topography and electrostatic properties have also been reported. These changes were dependent on the type of the plasma gas as shown in work by Cornelius et al. (2017) where $\mathrm{O}_{2}, \mathrm{CF}_{4}$, or $\mathrm{C}_{3} \mathrm{~F}_{6}$ gases were used on cellulose prior to papermaking and on the laboratory sheets. In a paper by a group of authors [Gonzaga et al., 2017) the analysis of elemental composition and identification of functional groups on the sample surface showed that chemical modifications occurred differently for each different plasma treatment. Contact angle measurements revealed that samples treated by $\mathrm{O}_{2}$ plasma remained hydrophilic, whereas low receptivity to polar $(\theta>$ $\left.120^{\circ}\right)$ and non-polar $(\theta>100$ ㅇ) liquids was observed for samples exposed to the SF6 plasma. Regarding plasma treatment of papers for print quality improvement Pykönen (2010) in his PhD made an extensive research regarding the use of plasma on offset printability of papers. With plasma activation, it was possible to increase the surface energy and hydrophilicity of paper. Both polar and dispersion interactions were found to increase, although the change was greater in the polar interactions due to induced oxygen molecular groups. The results indicated that plasma activation takes place particularly in high molecular weight components such as the dispersion chemicals used to stabilize the pigment and latex particles. Surface composition, such as pigment and binder type, was found to influence the response to the plasma activation. The ink-setting rate decreased with linseed-oil-based inks, probably due to increased acid-base interactions between the polar groups in the oil and the plasma-treated paper surface. With mineral-oil-based inks, the ink setting accelerated due to plasma activation. Hydrophobic plasma coatings were able to reduce or even prevent the absorption of dampening water into pigment coated paper, even when the dampening water was applied under the influence of nip pressure. In another paper by Touminen et al. (2010) different surfaces modifications of surfaces were investigated and the results showed that the traditional surface treatment methods, i.e., flame and corona treatments, increased the surface energy by introducing oxygen-containing functional groups on the surfaces of LDPE and PP more than helium and argon plasma treatments. Only in the case of flame treatment, the higher surface energy and oxidation level led to better print quality, i.e., toner adhesion and visual quality, than the plasma treatments. The morphological changes observed on LDPE surface after flame treatment are partly responsible for the improved print quality. Atmospheric plasma treatments improved the print quality of LDPE and PP surfaces more than corona treatment. The electret phenomenon observed on LDPE and PP surfaces only after corona treatment is the most likely reason for the high print mottling and low visual quality of corona treated surface. As the research of the plasma treatment of waxed papers is scars it motivated us to look further into the surface effects on these types of papers.

\section{METHODS}

We had three paper samples. One was the base paper which was waxed with two different type of wax compounds. The base paper had the grammage of $60 \mathrm{~g} / \mathrm{m}^{2}$ and specific volume of $1.50 \mathrm{~cm}^{3} / \mathrm{g}$ with a basic moisture content of $7 \%$. The main material is recycled fibres recovered from the deinking process which were combined with fibrillated recycled fibres. To evaluate the effectiveness of the waxing for barrier properties we have made the test to evaluate the water and oil absorption properties of the papers. The test was carried out before and after plasma treatment. The plasma treatment was carried out in laboratory conditions for $60 \mathrm{~s}$ with an atmospheric plasma laboratory unit $\left(\mathrm{O}_{2}\right)$ with the power of $500 \mathrm{~W}$ and $50 \mathrm{~Pa}$ pressure. The samples were tested before and after plasma treatment. For water resistance we have used several tests: the gravimetric Water vapour transmission rate (TAPPI T 464-om 18, with RH of 95\%), the ultrasound method for the determination of the liquid penetration dynamics (Emtec PDA unit and proprietary method) and contact angle measurements were made using water on the Fibrodat 1100 device. The test the oil resistance we have used the so-called KIT test which is based on the Tappi T 559 measures the degree of repellence or anti-wicking of paper and boards which have been treated with fluorochemical sizing agents used to prevent wetting of the cellulose fibres of the material. Test solutions with varying strengths of castor oil, toluene, heptane and turpentine are used. The highest numbered solution (the most aggressive) that remains on the surface of the paper without causing failure is reported as the "kit rating" (maximum 12). To further evaluate the effect of the plasma treatment we have tried to print the samples with water-based inks using flexo technology, where optical density was used to evaluate the possibility of printing. 


\section{RESULTS}

The results for the water transmission rate is presented in Figure 1.

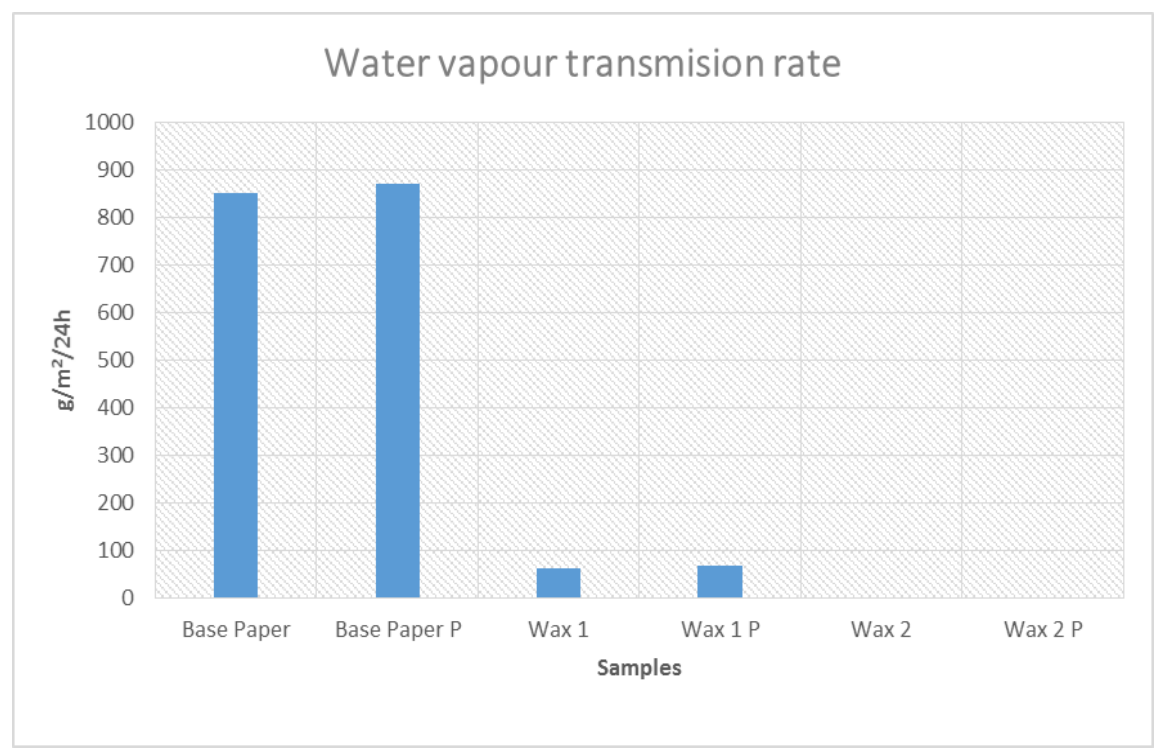

Figure 1: Water vapour transmission rate for the base and waxed papers

As we can observe as expected the non-waxed paper had a very high transmission rate $850 \mathrm{~g} / \mathrm{m}^{2} / 24 \mathrm{~h}$, while the waxed papers 1 had values $62 \mathrm{~g} / \mathrm{m}^{2} / 24 \mathrm{~h}$ and the waxed paper number 2 the lowest value of 8,7 $\mathrm{g} / \mathrm{m}^{2} / 24 \mathrm{~h}$. These were the results without the plasma treatment. After the plasma treatment there was a slight rise for the non-waxed base paper and the first waxed sample (from 850 to $870 \mathrm{~g} / \mathrm{m}^{2} / 24 \mathrm{~h}$ and 62 to $68 \mathrm{~g} / \mathrm{m}^{2} / 24 \mathrm{~h}$; while the third sample has dropped to $8 \mathrm{~g} / \mathrm{m}^{2} / 24 \mathrm{~h}$. These changes are very small due to the sensitivity of the test and we have tested the sample by the ultrasound measurement of penetration speed. The results are presented in Figure 2 and Table 1.

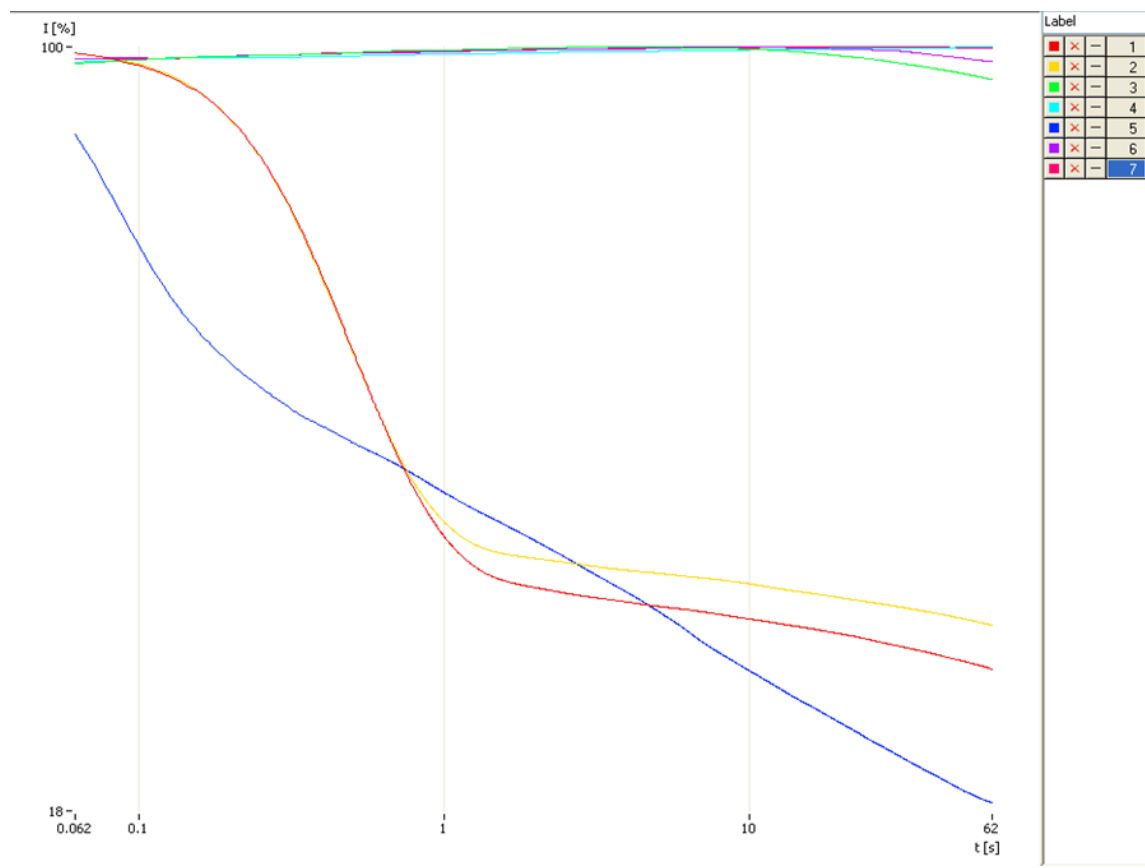

Figure 2: The penetration curves of water on different samples (curves 1 and 2 are base paper after plasma treatment, curve 3 wax 1 sample after plasma, curve 4 wax 2 after plasma and curve 5 base paper no plasma, curve 6 and 7 represent wax 1 and wax 2 sample with no plasma treatment) 
Table 1: Penetration parameters of the PDA measurement

\begin{tabular}{|l|l|l|l|l|l|l|}
\hline Sample & Base paper & Base paper $P$ & \multicolumn{1}{|c|}{ Wax 1 } & \multicolumn{1}{|c|}{ Wax 1 P } & Wax 2 & Wax 2 P \\
\hline Penetration & $\mathrm{Ci}=70,45$, & $\mathrm{Ci}=80,4$ & $\mathrm{Ci}=0,0$ & $\mathrm{Ci}=0,0$ & $\mathrm{Ci}=0,0$ & $\mathrm{Ci}=0,1$ \\
parameters & $\mathrm{Ct}=5,68$ & $\mathrm{Ct}=54,166$ & $\mathrm{Ct}=4,551$ & $\mathrm{Ct}=9,516$ & $\mathrm{Ct}=55,083$ & $\mathrm{Ct}=59,933$ \\
\hline
\end{tabular}

Figure 2 and Table 1 indicate that only the sample without wax (base paper) was influenced and had quicker absorption while the samples which were waxed had almost no penetration of water prior and after plasma treatment. This indicates that the surface pore structure has not changed in the macro-pore region and this could be negative to the printing properties based on water, but good for barrier properties. The water absorption and sorption properties were also checked with the contact angle measurement using Fibrodat 1100. The results are presented in Table 2.

Table 2: Contact angles of the samples

\begin{tabular}{|l|c|c|c|c|c|c|}
\hline Sample & Base paper & Base paper P & Wax 1 & Wax 1 P & Wax 2 & Wax 2 P \\
\hline $\begin{array}{l}\text { Contact angle } \\
\left(^{0}\right) \text { at 1s }\end{array}$ & 54,51 & 37,18 & 96,06 & 103,9 & 94,94 & 99,16 \\
\hline
\end{tabular}

The contact angle results also indicate that only the base paper had a decrease in contact angle (from $54,51^{\circ}$ to $37,18^{\circ}$ while the waxed papers had an increase in the contact angle measurements. This means that if we would like to print with water based inks the treatment does not bring any improvements.

As a final test before printing we have done the KIT test to test also the oil and grease repellency as this is an important property of the wrapping paper. The results are presented in Table 3.

Table 3: KIT values of the tested samples

\begin{tabular}{|l|c|c|c|c|c|c|}
\hline Sample & Base paper & Base paper P & Wax 1 & Wax 1 P & Wax 2 & Wax 2 P \\
\hline KIT number & $1 / 1^{*}$ & 1 & $9 / 7^{*}$ & 6 & $7 / 8^{*}$ & 5 \\
\hline
\end{tabular}

From Table 3 we can observe that here the plasma treatment caused some changes - lowered the oil and grease resistance of all samples. The base paper had the smallest KIT test value of 1 which means no barrier properties while the Wax 1 had the highest resistance value of 9 and 7 (for upper and downside) but decreased to 6 and sample Wax 2 decreased from $7 / 8$ to 5 . This means that the applied plasma treatment lowered the oil resistance of waxed papers by $1 / 3$ of the value. This is most probably due to the small scale of surface watching which opened up the micropore structure which is suitable for the oilbased liquid absorption into the porous network.

After testing the properties we have tried to print the samples. For printing we have used the IGT F1 flexo laboratory printer with printing parameters of printing speed of $0.5 \mathrm{~m} / \mathrm{s}$, anilox force to printing form $60 \mathrm{~N}$, printing pressure was $90 \mathrm{~N}$ and we have used HD flexo printing plates (1,4 mm thickness) and Doneck water-based inks with viscosity of $18 \mathrm{~s}$ (measured with ISO Cup 4).

The printing on the base paper was no problem and the plasma treatment had a beneficial effect regarding optical density and ink coverage as we can see in Figure 3.

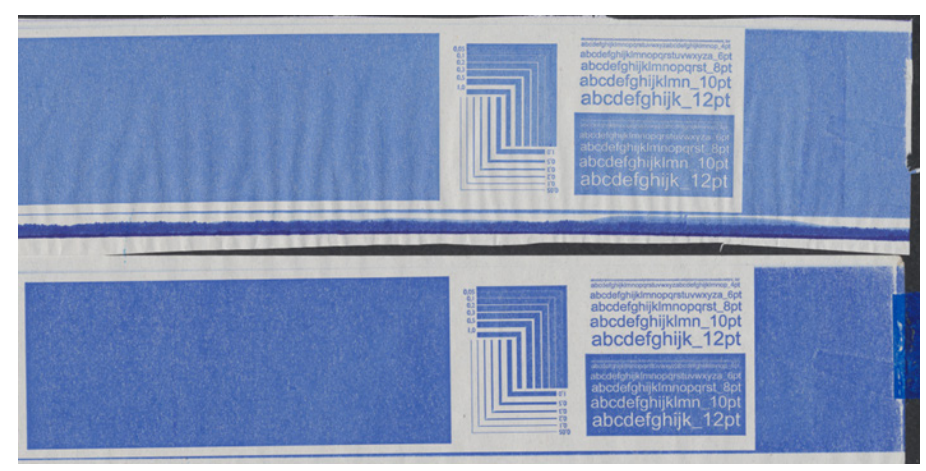

Figure 3: Print samples of the base paper (upper no plasma treatment lower after plasma treatment) 
The increase in the optical density was around 30\% for the plasma treated sample. No other print quality parameters were observed to change. The waxed paper on the other hand had a very poor printability as we can see in Figure 4.

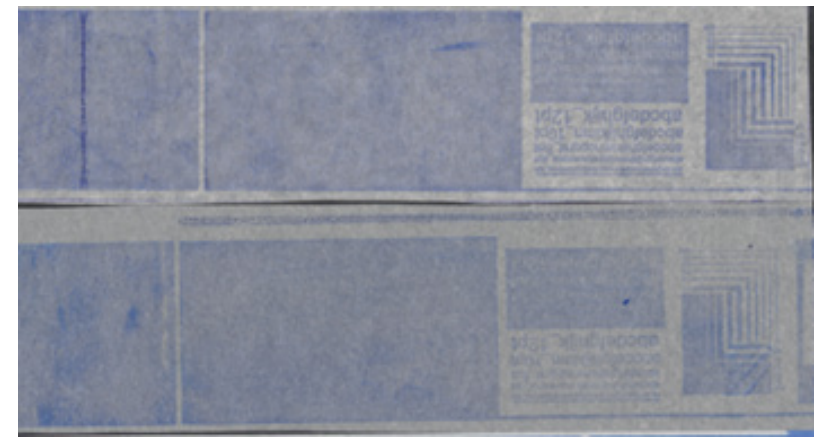

Figure 4: Print samples of the waxed paper (upper sample wax 2 lower sampler wax 1 sample before plasma treatment)

As we can see in Figure 4 the waxed paper are unsuitable for good quality prints in water-based flexo technology due to poor ink adhesion and coverage. After plasma treatment a very interesting effect had occurred which we present in Figure 5.

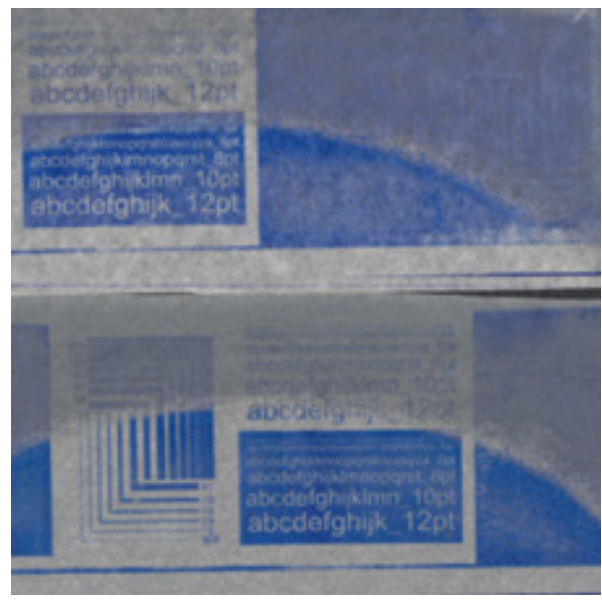

Figure 5: Prints of plasma treated samples

From Figure 5 we can observe two distinct surfaces with a poor coverage and with an excellent coverage. Bot surfaces were under plasma treatment with the exception that the spherical part of the sample which has been in the plasma treatment had also been in contact with the base plate which heats up. It seems that the thermal effect induced by the plasma treatment had a better printability effect on the samples from the surface modification effort.

\section{CONCLUSION}

Using waxing as a barrier coating properties enhancement has a long tradition and is widely used. The changes in the surface modification technologies like plasma or corona gives possibilities of printing food contact safe inks which are water-based. In this preliminary study, we tested several methods to determine the effect of atmospheric plasma on water and oil barrier properties as well the possible changes in flexo printability. Our results indicate that the applied amount of surface modification induced by plasma did not lower the water resistance (the water vapour transmission rate, the penetration dynamics and the contact angle were slightly changed but with no major effect), but lowered the oil and grease resistance by $30 \%$. The printability with water-based inks was not good, even after the plasma treatment which is in correspondence with the water/surface interaction test methods has shown. On the other hand as a very interesting phenomenon, the thermal effect within the plasma was observed 
where excellent printability was achieved. These structural and morphological changes will be tested in the fore coming period, as well as the optimization of the plasma application process.

\section{ACKNOWLEDGEMENTS}

The work was carried out within the RDI project Cel. Cycle: "Potential of biomass for development of advanced materials and bio-based products"(contract number OP20.00365), co-financed by the Republic Slovenia, Ministry of Education, Science and Sport and the European Union under the European Regional Development Fund, 2016-2020. The authors also want to thank Ita Junkar from the Jožef Štefan Insitute for the help with the plasma treatment.

\section{REFERENCES}

[1] Brunelli E.: "The potential of plasma technology", URL http://www.paperindustryworld.com/notonly- sustainable-the-potential-of-plasma-technology/ (last request 2018-10-07)

[2] Coles R. and Kirwan M. eds.: "Food and Beverage Packaging Technology", 2nd ed, (Blackwell Publishing Ltd, New Delhi, 2011), page 101.

[3] Cornelius, C., Saquing, C., Vendittti, R., McCord, M., Bourham, M.: "The Effect of Atmospheric Pressure Plasma on Paper and Pulps", BioResources, 12 (4), 8199-8216, 2017.

[4] Gonzaga de Camargoa, J.S., de Menezesa, A. J., da Cruzb, N.C., Rangelb, E.C., de Oliveira DelgadoSilvaa, A.: "Morphological and Chemical Effects of Plasma Treatment with Oxygen (O2) and Sulfur Hexafluoride (SF6 ) on Cellulose Surface", Materials Research, 20 (2), 842-850, 2017. doi: 10.1590/1980-5373-mr-2016-1111

[5] Kit L. Yam (Ed.): "The Wiley Encyclopedia of Packaging Technology", 3rd ed, (John Wiley \& Sons, 2010) page 910. doi: 10.1002/9780470541395

[6] National Research Council (U.S.). Food Protection Committee." Food-packaging Materials: Their Composition and Uses; a Report", National Academies, 1959

[7] Pykönen M.: "Influence of Plasma Modification on Surface Properties and Offset Printability of Coated Paper", PhD Thesis, Åbo Akademi University, Finland, 2010

[8] Sedlacekova Z.: "Food Packaging Materials Comparison of Materials Used for Packaging Purposes", Bachelor Degree International Business and Logistics Thesis, Helsinki Metropolia University of Applied Sciences, 2017.

[9] Tuominen, M., Lahti, J., Lavonen, J., Penttinen, T., Räsänen, P. J., Kuusipalo, J.: "The Influence of Flame, Corona and Atmospheric Plasma Treatments on Surface Properties and Digital Print Quality of Extrusion Coated Paper", Journal of Adhesion Science and Technology, 24 (3), 471-492, 2010. doi: $10.1163 / 016942409 \times 12561252292224$

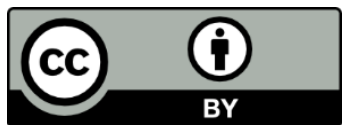

(C) 2018 Authors. Published by the University of Novi Sad, Faculty of Technical Sciences, Department of Graphic Engineering and Design. This article is an open access article distributed under the terms and conditions of the Creative Commons Attribution license 3.0 Serbia (http://creativecommons.org/licenses/by/3.0/rs/). 